$\xi=$ 圈

\title{
Electrostatic field theoretic approach to analyze the partial discharge phenomenon pertaining to insulation degradation
}

\author{
Shubhankan Mukherjee ${ }^{1 *}$, Adhir Baran Chattopadhyay ${ }^{1}$, Sunil Thomas ${ }^{1}$ \\ ${ }^{1}$ Department of Electrical and Electronics Engineering, BITS Pilani Dubai Campus, Dubai, United Arab Emirates \\ *Corresponding author E-mail: f20140205@dubai.bits-pilani.ac.in
}

\begin{abstract}
This paper elucidates the application of electrostatic field theory to analyze partial discharge due to the void formation inside the insulating material. Formation of voids leads to accumulation of static charges leading to capacitance build-up. The most probable cause of insulation failure is due to the subjection of high voltage. Prolonged high voltage poses a threat and leads to insulation failure. Failures occur in the tip gap between the conductor and insulating material's inner periphery. Probable causes of such failures are corona discharge, surface discharge and treeing, leading to formation of Lichtenberger figures in the material and cavity discharge. This paper presents a way of fabricating the inner lining of the insulator with a semiconductor layer obeying avalanche breakdown at breakdown voltage or voltages at which partial discharge is likely to occur. With the onset of high voltage which can cause a discharge, the semiconductor experiences avalanche breakdown giving out a single photon ejection by Geiger mode (principle). A superior prevention method of using Teflon for insulation instead of XLPE/PILC has been suggested and simulated using COMSOL. Detection using Avalanche photodetector(LiDAR) may enable us to track the probable location of the occurrence of partial discharge and isolate the system.
\end{abstract}

Keywords: Partial Discharge; Insulation Failure; Teflon Insulation; Avalanche Photodiode.

\section{Introduction}

Devices working on High Voltage sources face a constant threat of insulation degradation and failure. Stator winding insulation failure in Medium voltage AC motors leading to Line to Line fault or Line to Ground Fault was anlysed and predictive analysis to detect fault was stressed upon [1]. Now, in connection with stator winding insulation, over voltage and insulation coordination with continuous monitoring was possible using conditional monitoring by Finite element method [2]. Moreover, the effect of voltage, shape, size and protrusions on the conductor were studied using a simulation model based on Finite Element Analysis (FEA) software [COMSOL]. Three different types of void geometries were considered in this paper and their effect on partial discharge inception voltage, both in the presence and absence of protrusions, was studied[3]. Moreover tracing of dissected coils through visual inspection gave an idea of insulation failure [4]. Power cables are always under electrical stresses. Impact of static charges caused by dynamic electric fields [which are produced by ripples, polarity reversals, harmonics and transient switching pulses] leads to partial discharge phenomenon within the insulation and thus degrades the insulation. It was proved that PD greatly depended on dynamic electric field variations mostly due to inrush current[5]. Another form of stress is due to occurrence and utilization of Ultra High frequency (UHF). Two types of electrode configurations were considered for the analysis which were PointPlane and Rod-Plane Electrode. Stress developed due to UHF was calculated for various gap distances between the two electrodes, considering two configurations of electrodes for both Partial Discharge Inception Voltage (PDIV) and Breakdown Voltage
(BDV) [6]. The short-term insulation failure including the breakdown and surface flashover, and the employment of nanostructured fillers and surface treatments of polymers, were discussed in this paper. The sublimal effect of charge trapping and filling of voids were investigated using numerical methods and the transport of charge and movement behaviours in polymers based on surface potential decay and bipolar charge transport models were analysed. The deep trap effectively reduced carrier mean free path and the energy obtained from applied field [7].

Another cause of partial discharge is due to the presence of space charge. The accumulation of space charge in insulating (polymeric) materials and the effect of space charge on ageing rate under direct current, alternating current or impulsive voltage, directly through electric field alteration was discussed [8]. The effect of impulses on surface discharge of Polyethylene was shown by the change of PD numbers and phase-resolved PD patterns[9]. Different partial discharge (PD) models, namely the models of Gemant-von Phlippoff, Pedersen and Siemens were explained. Relationships were also offered between the apparent charge of a discharge appearing in enclosed cavities in solid dielectrics and the lifetime of a solid insulation as well as between the apparent charge and the sensitivity of PD detectors [10] [11]. Further, use of oil impregnated papers insulation was analysed [12]. It was found that it was easier for the oil-immersed power transformer to fail when moistened. Moisture may cause the great decrease of the breakdown voltage of the oil-paper insulation system [13]. It was further analysed that dieletric failure in insulation led to charge build-up and forming tiny capacitance[14] [15]. By applying the chopped sequence, more insight into the dielectric void was possible and allowed post partial discharge charge decay phenomena to be investigated [16]. Use of avalanche 
photodiode to detect partial discharge was taken into account to predict dielectric breakdown[17].

\section{Electrostatic field theory approach}

In this segment of analysis, we have used Maxwell's equation to define the degradation of insulators in the proximity of high voltage in devices such as current transformers windings, high voltage cable insulators and rotor windings.

At this juncture of our approach we first use the concept of void formation in materials. This can happen due to the following reasons:

1) Fabrication error

2) Continuous bombardment by electrons leading to wall enlargement and release of byproducts such as ozone and acidic compounds leading to degradation.

Void formation is, basically, due to partial discharge occurring in the high tension and high voltage cables. Void in non-viscous and solvent-less resin insulation is filled with gas or air having lower dielectric strength and permittivity in comparison to the surrounding insulating material.

Moreover, electrical stresses which is, basically, due to the high voltage and inrush current (at startup of motors). The electrical stress is electric field and is formulated below:

$$
E=\frac{U}{x}
$$

Considering the cable as coaxial with two or multiple concentric cylinders we can formulate the electrical stress as follows:

$$
\mathrm{E}=\frac{\mathrm{U}}{\mathrm{x} \ln \frac{\mathrm{r}_{0}}{\mathrm{r}_{\mathrm{i}}}}
$$

Where,

$\mathrm{U}=$ the source or applied voltage (forcing function) [in volts]

$\mathrm{x}=$ the radial distance to any point on the insulation [in millimeters]

$\mathrm{r}_{\mathrm{i}}=$ conductor radius [in milli-meters]

$\mathrm{r}_{0}=$ sheath radius [in milli-meters]

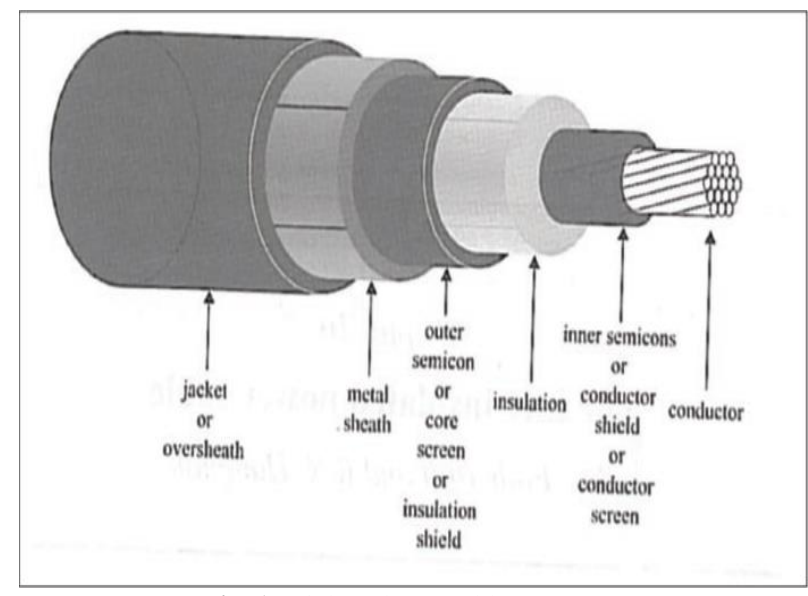

Fig. 1: High Voltage Cable Structure.

(C) by Prof. Nelson Ljumba, University of KZN

Now, if we consider integral form of Gauss' law for electrical field considering in spherical coordinate system we formulate the closed surface integral to be as follows:

$$
\oint_{S} \vec{E} \circ \hat{n} d a=\frac{q_{\mathrm{enc}}}{\varepsilon_{0}}
$$

$\mathrm{E}=$ Electric field $(\mathrm{V} / \mathrm{m}), \mathrm{q}=$ charge enclosed (coulomb), $\varepsilon_{0}=$ permittivity in vacuum, $n=$ unit vector perpendicular to the plane of the surface area, da = elemental surface area and the symbol for integration stands for closed surface integral

Now, we focus on important parameters responsible for partial discharge. The parameters in our purview here is charge and dielectric strength of the insulation (material).

Partial discharge is related to the discharge of excess charge leading to breakdown. So excessive charge builds up in the void leads to a formation of pseudo capacitance.

\section{Lumped capacitance model for void for- mation and partial discharge}

We used the lumped capacitance or 'ABC model' to model the formation of capacitance in voids due to material non linearities.

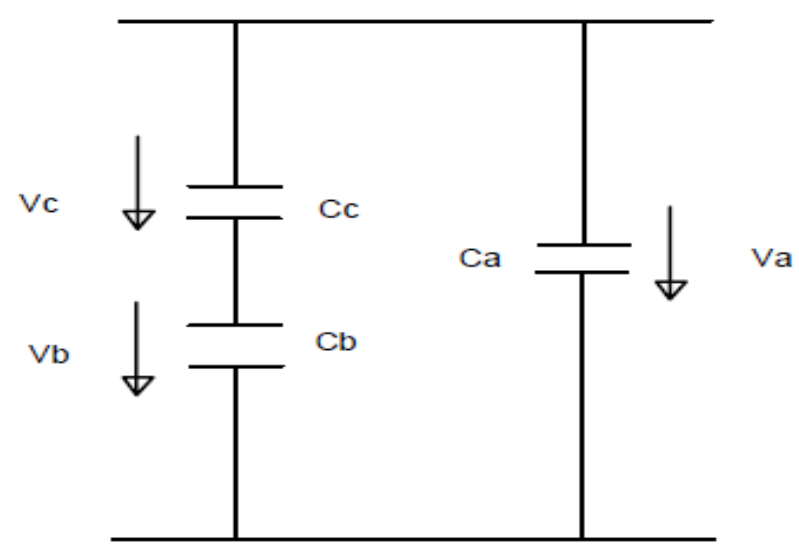

Fig. 2: Capacitance Model Mimicking a Void Formation.

Whenever there is a void and charge build-up, it is like a capacitor in parallel to the circuit capacitance. In case of multiple voids, the capacitors act as a series capacitor bank in parallel with the circuit capacitance.
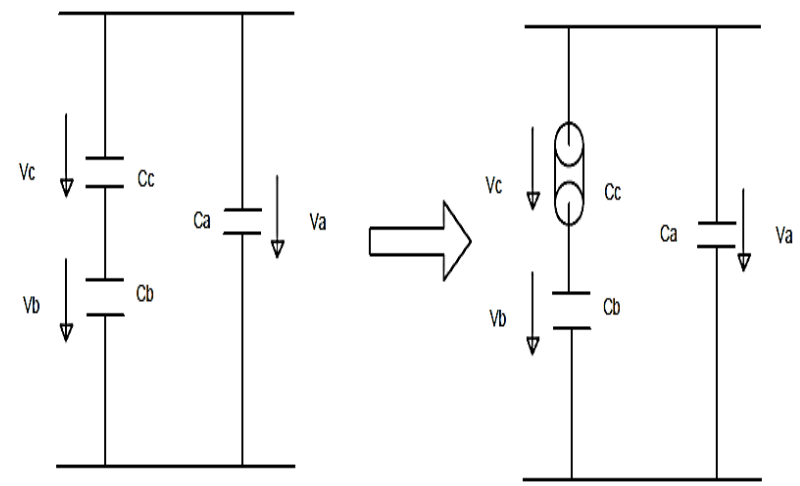

Fig. 3: ABC Model of a Void Formation.

An enclosed cavity or void inside a solid insulation can be modeled as a capacitance and we name it as $\mathrm{C}_{\mathrm{c}}$. The insulation capacitance next to the void as $C_{b}$ and rest of the capacitance as $C_{a}$ which is known as the circuit insulation capacitance, or the overall capacitance.

The voltage drop across the void or cavity by voltage division rule is given by,

$\mathrm{V}_{\mathrm{c}}=\mathrm{V}_{\mathrm{a}} \frac{\mathrm{C}_{\mathrm{b}}}{\mathrm{C}_{\mathrm{b}}+\mathrm{C}_{\mathrm{c}}}$

The apparent charge stored in the void (capacitor) responsible for the discharge is given by,

$\mathrm{q}_{\mathrm{a}}=\mathrm{C}_{\mathrm{b}} \Delta \mathrm{V}_{\mathrm{c}}$

Where,

$\Delta \mathrm{V}_{\mathrm{c}}=$ is the voltage drop across the void (in volts) 
Now, we consider the line charge density on a single plate and volume charge density for the void considering it to be spherical in nature.

Linear charge density, $q_{\mathrm{enc}}=\lambda L$

Volume charge density, $q_{\mathrm{enc}}=\rho V$

The charges confined in the void is directly linked to the origin of electric flux, and the free charges lead to development of electric flux density (D).

$$
\vec{\nabla} \circ \vec{E}=\frac{\rho}{\varepsilon_{0}}
$$

As $\mathrm{E}=-\vec{\nabla} \mathrm{V}$, it yields that,

$$
\nabla^{2} V=-\frac{\rho}{\varepsilon}
$$

As a part of Maxwell's equation set, it may be reminded that

$$
\mathbf{D}=\varepsilon \mathbf{E}
$$

$\nabla \cdot \mathbf{D}=\rho_{V}$

The presence of this apparent charge $(\rho)$ can be useful for the detection of Partial Discharge pulses.

\section{Experimental setup for the measurement of partial discharge}

In order to determine the cavity discharge and current spike for an insulator, the experiment was performed by Gemant and Philippoff setup as shown in Fig 4. And the concerned experimental result is shown as the distorted waveform in Fig.5.

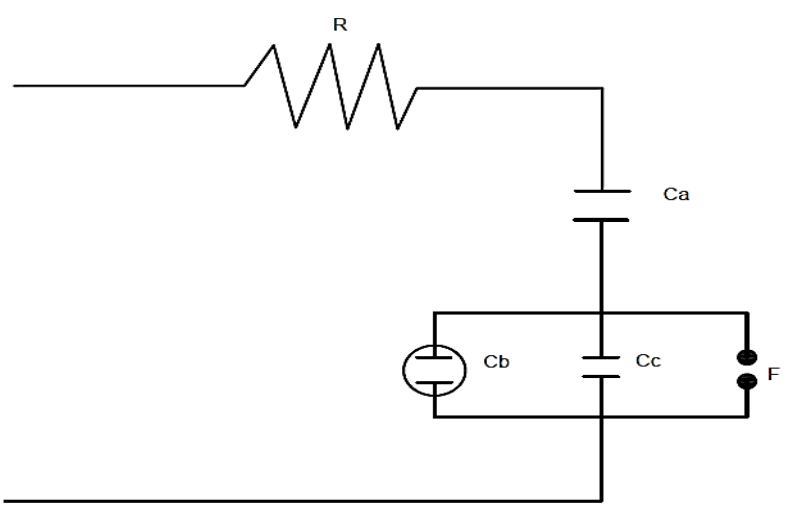

Fig. 4: Experimental Setup with Spark Gap F.

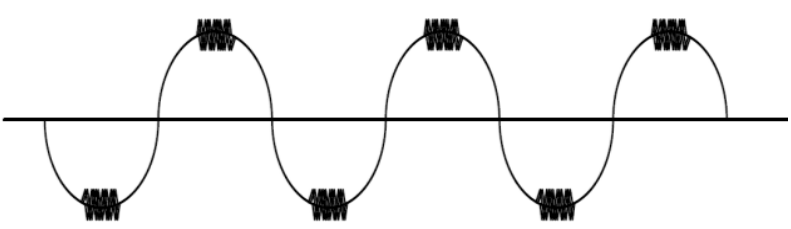

Fig. 5: Partial Discharge Pulses in Sinusoidal AC Signal.

This model used the theory of breakdown and spark gap current during the discharging time in cavity due to capacitance Cc. But the current $i_{b}$ due to the capacitance $C_{b}$ also flows through the test object as in case of capacitance Cc causing ambiguity in the measurement.

So, on validating the apparent external void charge we arrive on the following equations,

$\mathrm{q}_{\mathrm{a}}=\Delta \mathrm{V}_{\mathrm{c}}\left(\mathrm{C}_{\mathrm{b}}+\mathrm{C}_{\mathrm{c}}\right)$
We also know that the external charge is the time integral of the current flowing through the capacitances $C_{b}$ and $C_{c}$, so we arrive at the final equation,

$\mathrm{q}_{\mathrm{a}}=\mathrm{q}_{\mathrm{c}} \frac{\mathrm{C}_{\mathrm{b}}}{\mathrm{C}_{\mathrm{b}}+\mathrm{C}_{\mathrm{c}}}$

But measurement of discharge current is not feasible and a more accurate way to measure is the dipole moment due to the dielectric polarization in the voids as due to partial discharge there is formation of secondary elements like ozone and acidic compounds having greater relative permittivity and thereby leading to increase in capacitance. Fig 4 can be modified as shown in Fig 6, showing indication of current distribution.

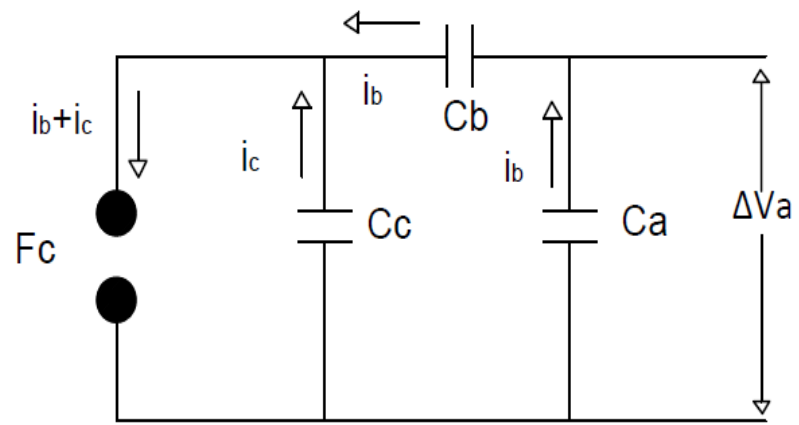

Fig. 6: Characteristic Circuit Elements of Capacitive PD Model

As in a capacitor, due to the existence of electric field and it finally leads to development of displacement current according to Maxwell's postulate.

$\nabla \times \mathbf{H}=\frac{\partial \mathbf{D}}{\partial t}+\mathbf{J}$

$\mathrm{H}=$ Magnetic field [Tesla], $\mathrm{D}=$ Electric Flux density [coulomb/sq.m], J= current density [amperes per square metre]. Whenever partial discharge phenomenon occurs in an insulator material, discontinuity in charge distribution takes place and it leads to the development of current spikes. This is portrayed in Fig.5.

For our mathematical simplicity, we will normalize the displacement current equal to the charging current across the void and it can be written as,

$\mathrm{I}_{\mathrm{D}}=\mathrm{I}_{\mathrm{ch}}=2 \pi \mathrm{fCU}$

Where

$\mathrm{f}=$ Supply frequency (Hertz), $\mathrm{C}=$ capacitance of the void (Farads), $\mathrm{U}=$ supply voltage (volts)

For a coaxial cable the capacitance can be written as follows,

$$
C=\frac{2 \pi \varepsilon_{0} \varepsilon_{r}}{\ln \frac{r_{o}}{r_{i}}}
$$

We can measure the current variation at macroscopic level relative to the entire length of a cable to detect through partial discharge pulses on oscilloscope or the electric field variation due to dielectric suppression through Electromagnetic or Dielectric spectroscopy in a Bode or Nyquist plot.

\section{Alternative approach to reduce the partial discharge in high voltage cables}

The current technology uses Cross Linked Polyethene (XLPE) cables and Paper Insulated and Lead Covered cables (PILC) cables. 
Since cable capacitance is so important, a lot of analysis goes into minimizing it, but the hypothesis is not being adaptive due to the following reason.

1) Increasing the insulation wall thickness which will lead to additional material cost.

2) Decreasing the conductor diameter but it will increase the skin effect at higher frequencies.

The PTFE (Teflon) films used as an insulation layer is quite robust and the dielectric strength is a function of the film thickness, which is defined by the following relationship,

$\mathrm{E}_{\mathrm{B}} \alpha \mathrm{T}^{-\mathrm{k}}$

Where $\mathrm{E}_{\mathrm{B}}$ is the dielectric strength, ' $\mathrm{T}$ ' the film thickness, and $\mathrm{k}$ is the fitting parameter. It is to be noted that the $\mathrm{k}$ factor can be varied in accordance with the cable configuration, internal microscopic structure and charge transfer rate within the insulating Teflon material. As indicated by the relationship, the dielectric strength is inversely proportional to the thickness and dielectric strength decreases with increasing thickness.

Another factor which adds onto external stress on insulating material, subjected to high voltage, in regard of the charge distribution, is thermal energy. As the temperature rises due to the heating effect of current, the electrons accelerate further leading to lowering of the insulating material's dielectric strength. At very low temperature, all charged particles are trapped and are closely held together. As the temperature rises, the trapped electrons will get delocalized more easily, and less electric field is needed.

Moreover, with the increase of temperature the overall volume increases, and the dielectric strength decreases with the decrease of the material density, Thus, it is expected that $\mathrm{E}_{\text {в }}$ decreases with increasing temperature. This proves that the dielectric strength decreases as the temperature increases and with the increasing material volume due to thermal effect.

According to the formula of capacitance, out of the three parameters, we have one last parameter to overcome the issue of Partial Discharge is by using an insulation with lower dielectric constant.

$\mathrm{C}=\frac{\mathrm{Q}}{\mathrm{V}}=\frac{\mathrm{Q}}{\mathrm{Ed}}=\frac{\mathrm{A} \epsilon}{\mathrm{d}}$

$\mathrm{C}=$ Capacitance (Farads), $\mathrm{Q}=$ Charge (coulomb), $\mathrm{E}=$ Electric field $(\mathrm{V} / \mathrm{m}), \mathrm{D}=$ plate distance $(\mathrm{m}), \mathrm{A}=$ Area $\left(\mathrm{m}^{2}\right), \in=$ Relative permittivity.

Since the partial discharge occurs in the tip gap separation of the conductor and insulator, we can sandwich the gap of the conductor and insulator with a material having lower relative permittivity such as Teflon sheet as it has higher dielectric strength.

This new Teflon technology will prove better than XLPE and PLIC cables having following characteristics.

Table 1: Data for Different HV Cables

\begin{tabular}{lll}
\multicolumn{3}{c}{ Table 1: Data for Different HV Cables } \\
\hline Material Type & $\begin{array}{l}\text { Relative Permit- } \\
\text { tivity }\end{array}$ & $\begin{array}{l}\text { Dielectric } \\
\text { Strength }\end{array}$ \\
\hline $\begin{array}{l}\text { Teflon With Benzene Impreg- } \\
\text { nated Paper }\end{array}$ & 2.02 & 162 \\
XLPE & 2.23 & 145 \\
PILC & 3.76 & 90 \\
\hline
\end{tabular}

Along with that, we can coat the insulator side with a semiconducting layer (Silicon Carbide) which will reduce the possibility of air gap or void formation leading to the reduced possibility of a spark gap or discharge. As our whole discussion concentrates on the localization of charges on an insulating material, at this stage it demands for portraying a model of the H.V cable and it is shown in Fig.7

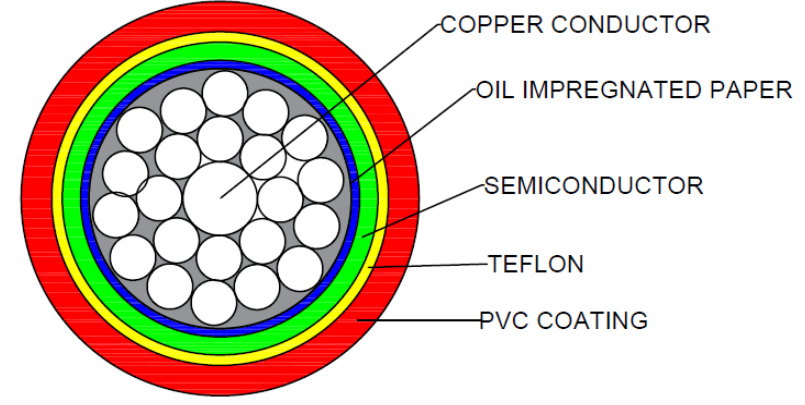

Fig. 7: Cross Sectional View of Proposed HV Cable Model.

With the prevalent stage of technology, we can detect in-situ Avalanche breakdown. We can measure the single photon electromagnetic wave using Geiger counter to trace any unwanted partial discharge and isolate the material before any degradation or faults [Fig.8].

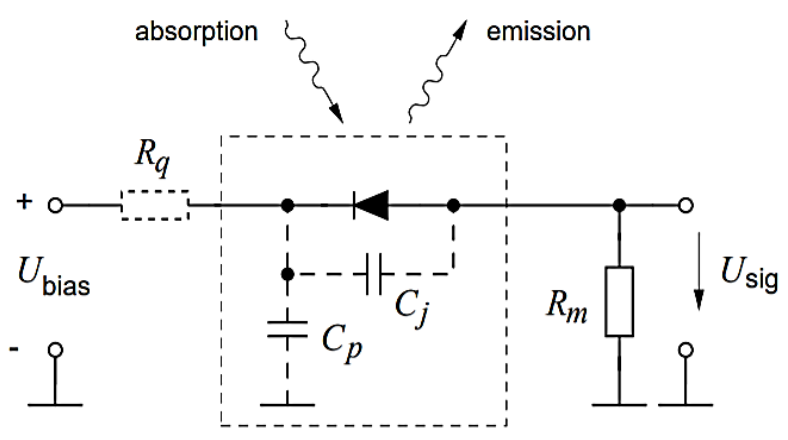

Fig. 8: A) Experimental Setup for Photon Detection.

(C) Christian Kurtsiefer, Patrick Zarda, Sonja Mayer, and Harald Weinfurter [18]

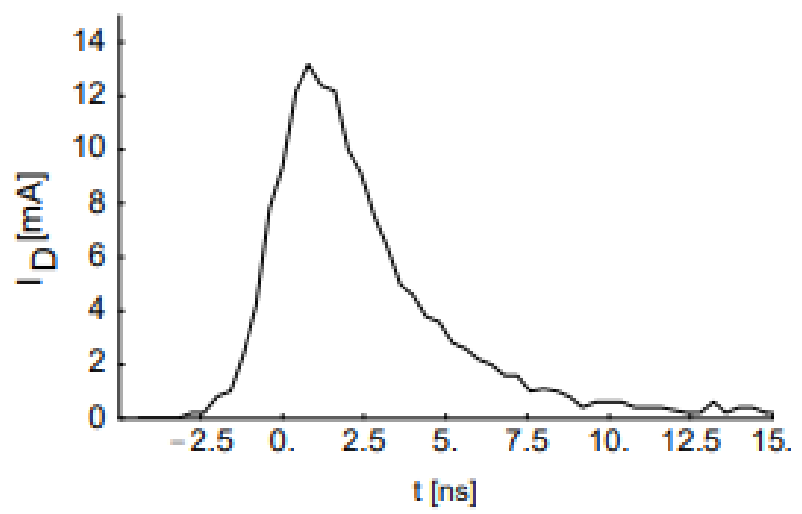

Fig. 8: B) Discharge Current of the Photodiode during A Breakdown Cycle

The experiment performed by Christian Kurtsiefer, Patrick Zarda, Sonja Mayer, and Harald Weinfurter is shown in Figure 8(a). In this system the diode has been modelled by a junction capacitance and parasitic capacitance. When the photodiode faces an avalanche, breakdown discharge current flows and its experimental plot as a factor of time as reported by the authors is shown in figure 8(b)

In this current research methodology, the avalanche breakdown, having a spectral emissivity of about $1.1 \mu \mathrm{m}$ to $2.1 \mu \mathrm{m}$ during the avalanche breakdown has been simulated through electric and magnetic field dynamics using COMSOL. As these wavelengths can travel through the insulators, detection of a partial discharge becomes easier. So, with the help of infrared spectroscopy, we can detect photons when there is partial discharge due to breakdown owing to high/over voltage. On finding out the exact location of the PD we can isolate the system and prevent from further damage. 


\section{Result and conclusion}

In this paper we were able to map the relation between partial discharge and the theoretical aspects of electrostatic field and find out the root cause of partial discharge due to the formation of capacitance in the void due to excess apparent charge which occurs due to high voltage originating from electric field due to charge localization.

On having found out the basic analogy which causes material degradation, we were able to adopt a better insulating material such as Teflon having higher discharge strength and lower relative permittivity. Apart from that, this paper devises an innovative technology of using Silicon Carbide avalanche diode to be layered between the Teflon and oil impregnated paper to make Partial Discharge and void formation trackable and prevent any early onset of fault.

By using COMSOL simulations, following conclusions are drawn. COMSOL simulations for the above-said analysis was performed with the following data.

The simulations were synthesised at a voltage of $33 \mathrm{kV}$ (Medium voltage) volts with electric displacement field of $30 \mathrm{C} / \mathrm{m}^{2}$, short circuit current of $330 \mathrm{~A} / \mathrm{m}$ and surface charge density of $300 \mathrm{C} / \mathrm{m}^{2}$. Single core conductor radius was 50 milli-meter and other layers were placed at a gradation of 25 milli-meters apart from the conductor. The following plots were obtained.

i) Electric Field due to electrostatic charges located in the conductor. In the simulation, electric field is particularly visible in dielectric materials like paper and Teflon. Maximum field strength is concentrated on the inner core of the conductor as per the simulations due by direct application of Gauss' Law in Electrostatics.

$\mathrm{E}=\mathrm{K} \frac{\mathrm{Q}}{\mathrm{r}^{2}}$ And $\mathrm{E}=\frac{\mathrm{dV}}{\mathrm{dr}}$

ii) As the radius of the conducting sphere decreases the electric field concentration increases or in other words the magnitude becomes greater. The peak value of electric field as per simulation is $9.7 \times 107 \mathrm{~V} / \mathrm{m}$ and the minimal value is found to be $-1.03 \times 108 \mathrm{~V} / \mathrm{m}$ as shown in figure 9 . In this simulation the electric field existence is in the dielectric material like paper and Teflon.
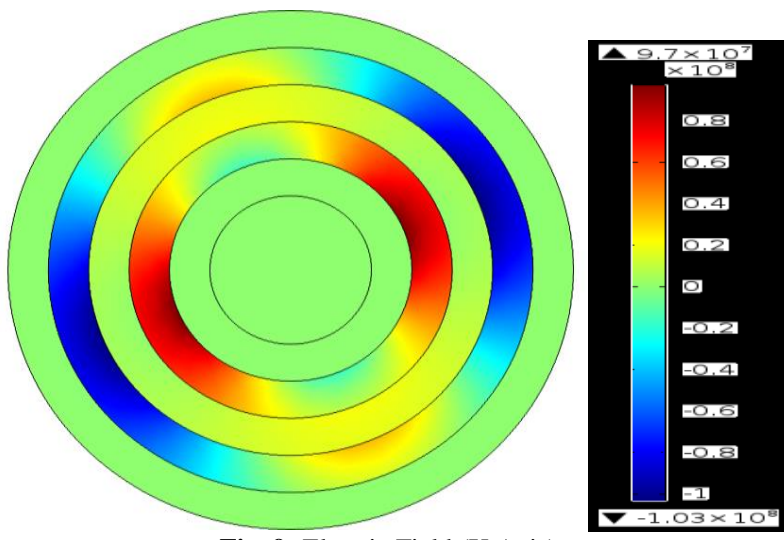

Fig. 9: Electric Field (X Axis).

iii) Most of the electrical insulators have electrons densely packed to their nucleus. When an electric field or potential is applied, the negative charge cloud shifts from the positive charge cloud and realigns. This leads to the decrease of net electric field.

$\mathrm{E}_{\text {net }}=\mathrm{E}-\mathrm{E}_{\text {polarisation }}$

This polarisation leads to the formation of capacitance (charges in voids) due to charge build-up in the voids due to overtime material degradation. As per our simulation the surface polarization was found to be in the range of $6.43 \times 10^{6}$ to $-6.44 \times 10^{6}$ as shown in figure 10 .

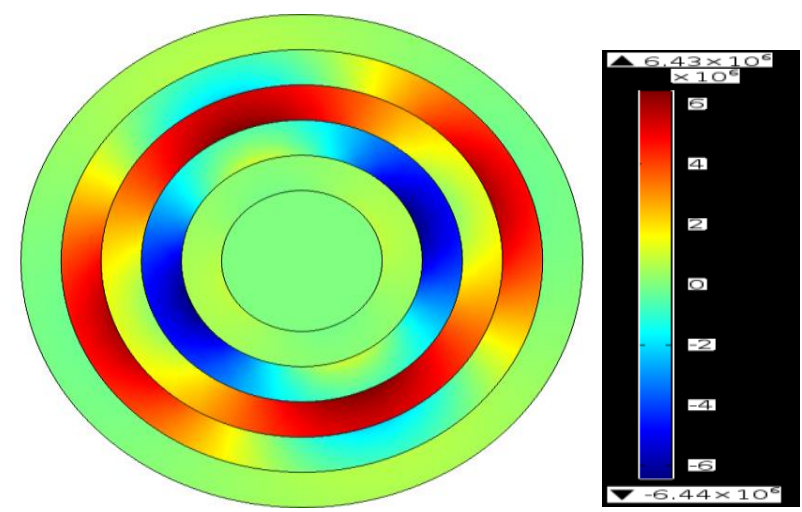

Fig 10: Polarisation Due to Electric Field.

iv) On account of electric field arising due to static charges, in a high voltage cable, most of the static charges originates on the tip gap between the current carrying conductor and the insulating material. So, the maximum electric field (normal) to the surface is found near the tip gap is $1.18 \times 108 \mathrm{~V} / \mathrm{m}$ and the minimal value is found to be $3.94 \times 10-9 \mathrm{~V} / \mathrm{m}$ as shown in figure 11.

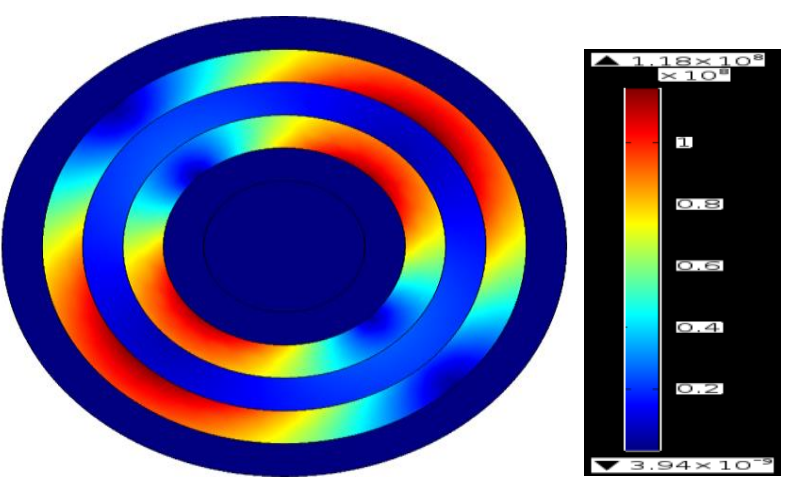

Fig. 11: Electric Field (Normal).

v) Now the dynamic conditions of current flowing inside a conductor leading to the formation of magnetic field due to magnetomotive force is considered. This MMF is used by clamp meters to measure current which is basically a current transformer with the cable being the primary core and the clamp meter being the secondary core. Applying electromagnetic field theory, we find that the magnetic flux density can be related to magnetic field in the following manner,

$\mathrm{B}=\mu \mathrm{H}$

Where $B$ is the magnetic flux density, $\mu$ is the permeability of the material and $\mathrm{H}$ being the magnetic field. In our simulations we find the magnetic field has maximum appearance near the outer insulation material as they form a closed loop as per Maxwell's equation. The above phenomenon becomes more prominent in the semiconductor material when it encounters short circuit current leading to avalanche breakdown due to collision of electrons. Here we apply Amperes law into study where the curl of magnetic field is given by

$$
\nabla \times \mathbf{H}=\mathbf{J}+\frac{\partial \mathbf{D}}{\partial t}
$$

$\mathrm{H}=$ Magnetic field (Tesla), $\mathrm{J}=$ Current Density (A/sq.m) and $\mathrm{D}=$ Electric flux density (coulomb/sq.m).

Which is influenced by both magnetic field and electric field. As per our simulations, we find the magnetic flux density to be in the range of 0.87 to $1.97 \times 10^{-11} \mathrm{~Wb} / \mathrm{m}^{2}$ (or Tesla) as shown in figure 12 


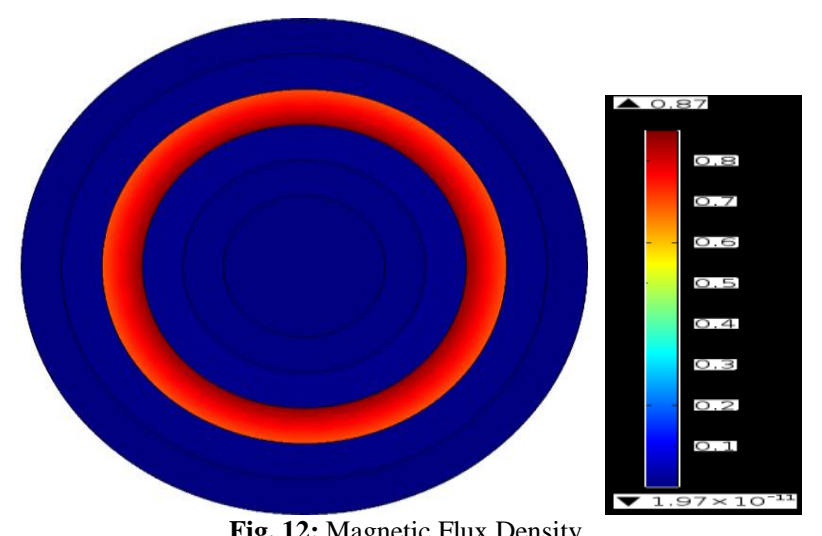

Fig. 12: Magnetic Flux Density.

(vi)The final stage of this research work is to find the early insulation degradation of insulating material. Due to high voltage transmission or conduction, the materials are under constant electrical stress. Now in cross electric and magnetic field with magnetic field into our picture, under dynamic conditions the polarisation effect upsurges. As per simulation the net polarisation was found to be $2.01 \times 10^{5}$. Due to high voltage most common causes of failure are treeing(Lictenberger figures), surface corona discharge and charge accumulation in void. As mentioned in figure 13 , figure 14 and figure 15 respectively, due to high surface current density $\left(2.8 \times 10^{5}\right.$ to $\left.4.35 \times 10^{-8}\right)$, high relative permeability $\left(33 \times 10^{5}\right.$ to 0.25$)$ and high voltage $(3.3 \mathrm{kV})$, there is external current density due to charge build-up in the tip gap layer between semiconductor and teflon insulation layer leading to avalanche breakdown of the silicon carbide semi-conductor due to collision of electrons which leads to generation of single photon. Moreover electric field plays an important role leading to Zener breakdown of the dielectric material namely the paper insulation and teflon layer. This can be detected with photo-detector and any fault like winding short (line to line or line to ground) or flash over in transmission lines due to insulation failure can be detected and rectified at primitive stage.

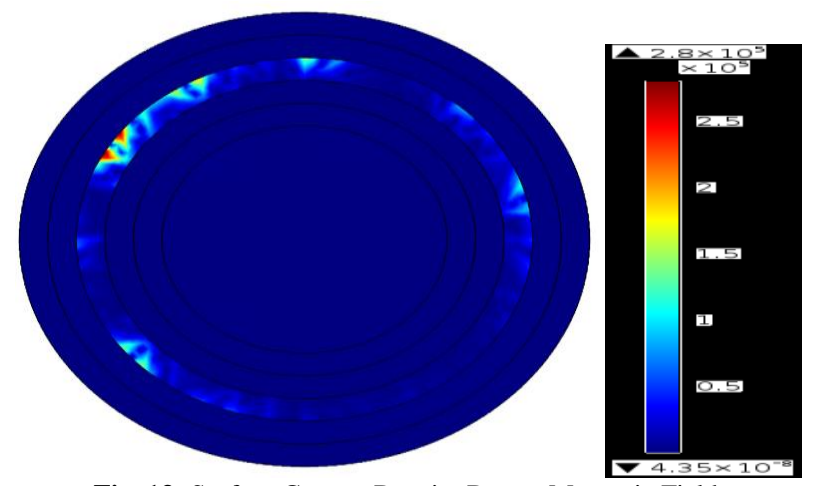

Fig. 13: Surface Current Density Due to Magnetic Field.
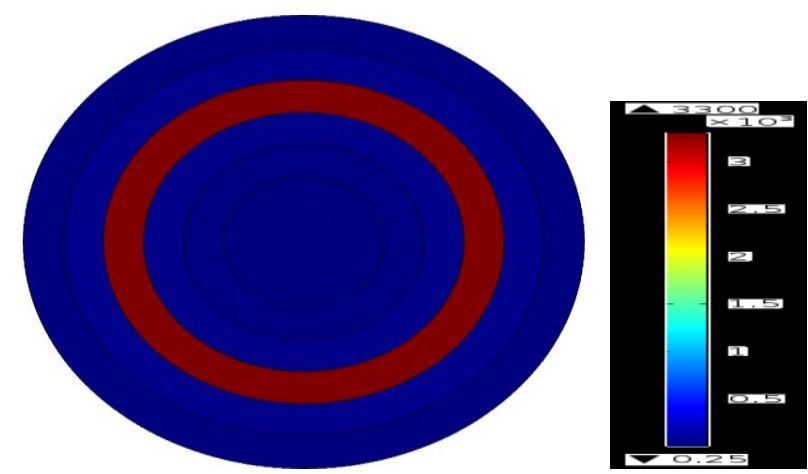

Fig. 14: Relative Permeability.

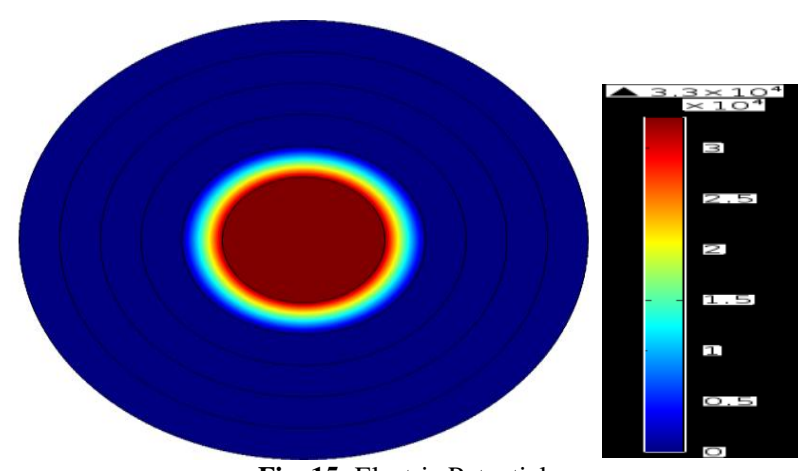

Fig. 15: Electric Potential.

\section{References}

[1] Sang Bin Lee, Tae-June Kang, Heedong Kirn, Taesik Kong and Chaewoong Lim, "CASE STUDIES OF STATOR WINDING TURN INSULATION FAilURES INMEDIUM VOLTAGE MOTORS", IEEE Pulp, Paper And Forest Industries Technical Conference (PPFIC), 2017

[2] X. Li, P. Zhou, H. He and L. Wang, "Study of overvoltage and insulation coordination of UHV DC transmission line," 2017 1st International Conference on Electrical Materials and Power Equipment (ICEMPE), Xi'an, 2017, pp. 354-358. https://doi.org/10.1109/ICEMPE.2017.7982102.

[3] Sambit Hore, Souryadeep Basak, Nasirul Haque, Sovan Dalai, Monish Mukherjee, "Studies on the Effect of Void Geometry and Location on Electric Field Distribution and Partial Discharge in XLPE Insulated Power Cable by Finite Element Analysis using COMSOL Multiphysics Simulation" 2017 6th International Conference on Computer Applications In Electrical EngineeringRecent Advances (CERA). https://doi.org/10.1109/CERA.2017.8343330.

[4] Anna Gegenava, Dr. Aleksandr Khazanov, Bill Moore, P.E, "Failure Evaluation of the Electrical Insulation for High Voltage Stator Windings Machines and Root Cause Analysis through Visual Inspection ofDissected Coils", IEEE 2016 Electrical Insulation Conference (EIC), Montréal, Qc, Canada, 19 - 22 June 2016.

[5] M. Azizian Fard, M. Emad Farrag, S. G. McMeekin and A. J. Reid, "IEEE Transactions on Dielectrics and Electrical Insulation", Volume: 24, Issue: 3, p. 1494 - 1502, June 2017.

[6] Karthik B G , Kavya C.S, Prasanta Kundu, " Analysis of electric stress and partial discharge Ultra High Frequency signal”, 2017 3rd International Conference on Condition Assessment Techniques in Electrical Systems (CATCON).

[7] Shengtao Li, "Charge Dynamics:Linking Traps to Insulation Failure", 2015 IEEE 11 th International Conference on the Properties and Applications of Dielectric Materials (ICPADM).

[8] Gian Carlo Montanari, "Bringing an Insulation to Failure: the Role of Space Charge", IEEE Transactions on Dielectrics and Electrical Insulation Vol. 18, No. 2; April 2011. https://doi.org/10.1109/TDEI.2011.5739438.

[9] Roya Nikjoo, Nathaniel Taylor, Hans Edin, "Effect of High Voltage Impulses on Surface Discharge Characteristics of Polyethylene", IEEE 24th Nordic Insulation Symposium on Materials, Components and Diagnostics. https://doi.org/10.5324/nordis.v0i24.2291.

[10] Michael G. Danikas - Grigorios E. Vassiliadis, "models of partial discharges (PD) in enclosed cavities in solid dielectrics: a study of the relationship of pd magnitudes to the sensitivity of pd detectors and somefurther comments on insulation lifetime", Journal of ELECTRICAL ENGINEERING, VOL. 54, NO. 5-6, 2003, 132 135.

[11] E. Lemke, "A Critical Review of Partial-DischargeModels", IEEE Electrical Insulation Magazine ( Volume: 28, Issue: 6, Nov.-Dec. 2012 ).

[12] Dr. Ir. J. Vermeer and Ir. W. Boone, "Characteristic properties of oil-impregnated laminatedsynthetic insulation for extra-highvoltage cables", Proceedings of the Institution of Electrical Engineers ( Volume: 114, Issue: 7, July 1967 ).

[13] Wenxiong Mo, Huaying Bai, Yangchun Cheng, Zhenliang Zhang, Danqing Huang, Chongzhi Zhao, Dan Li, "A Failure Probability Model of the Moistened Oilpaper Insulation of Transformers Based on Strengthstress Model", 2016 IEEE International Conference on High Voltage Engineering and Application (ICHVE). https://doi.org/10.1109/ICHVE.2016.7800697. 
[14] D. Tommasini, "Dielectric insulation and high-voltage issues", CERN- 2010-004, pp. 335-355.

[15] Prof. Nelson Ljumba, "High voltage cable insulation systems", energize - May 2008.

[16] Marek Florkowski, Barbara Florkowska and Romuald Włodek, "Investigations on Post Partial Discharge Charge Decay in Void Using Chopped Sequence", IEEE Transactions on Dielectrics and Electrical Insulation Vol. 24, No. 6; December 2017. https://doi.org/10.1109/TDEI.2017.006625.

[17] B. F. Levine and C. G. Bethea, "Single photon detection at 1.3,urn using a gated avalanche photodiode", Appl. Phys. Lett. 44, 553 (1984). https://doi.org/10.1063/1.94800

[18] Christian Kurtsiefer, Patrick Zarda, Sonja Mayer, and Harald Weinfurter, "The breakdown flash of Silicon Avalance Photodiodes backdoor for eavesdropper attacks?" , Journal of Modern Optics 48(13):2039-2047. 